\title{
REDES DE COLABORACIÓN ACADÉMICA: EL CASO DE LAS COAUTORÍAS DE ARTÍCULOS PRESENTADOS EN LA CONFERENCIA ACADÉMICA PERMANENTE DE INVESTIGACIÓN CONTABLE -CAPIC- $(1993-2015)^{1}$
}

\author{
ACADEMIC COLLABORATION NETWORK: \\ THE CASE OF CO AUTHORSHIPS OF THE \\ ARTICLES PRESENTED AT THE PERMANENT \\ ACADEMIC CONFERENCE ON RESEARCH \\ ACCOUNTING -CAPIC- CHILE, (1993-2015)
}

\author{
Luis Jara Sarrúa², Ángelo Benvenuto Vera³ ${ }^{3}$ Ricardo Méndez Romero ${ }^{4}$
}

\section{RESUMEN}

En la actualidad, el fomento a la producción científica universitaria se centra en la creación de redes de colaboración. En este sentido, en el año 1990 se creó la sociedad científica Capic-Conferencia Académica Permanente de Investigación Contable-, institución que alberga, principalmente, a académicos adscritos a universidades chilenas, tanto públicas como privadas. Bajo este contexto, el propósito de esta investigación se relaciona con identificar en qué medida la participación de investigadores en la Capic, durante el período 1992-2015, ha generado redes de colaboración, así como identificar los principales atributos y las estructuras internas de dichas redes. Los hallazgos demuestran que la macro red generada durante dos décadas posee baja cohesión, con alta fragmentación, y un predominio del género masculino. Desde una perspectiva micro, el análisis de las ocho principales redes evidencia un predominio de ciertas universidades, siendo la micro red con mayor integrantes la configurada principalmente, por académicos de la Pontificia Universidad Católica de Valparaíso. Además, se observa que posiciones relevantes en torno a la conexión y la cercanía con otros investigadores son asumidas por mujeres; aun cuando en la mayoría de las redes predomina el género masculino. Por último, otro resultado se asocia a la existencia de una micro red conectada internacionalmente, en concreto con investigadores de Colombia, Argentina, y Chile. Futuras investigaciones deben profundizar en identificar los capitales de las redes con la finalidad de visualizar y gestionar dichos recursos -económicos, intelectuales, culturales, sociales-; para fomentar la conectividad entre investigadores con intereses comunes, facilitando la localización de redes con ventajas estructurales que fomenten una mayor productividad entre los académicos que participan en la Capic.

Palabras claves: Análisis de redes sociales, redes de investigación, coautoría, Capic, contabilidad.

Recepción: 24/11/2016. Aprobación: 05/10/2017.

1 Proyecto presentado por los académicos: Ángelo Benvenuto V., Luis. Jara S. y Ricardo Méndez R., al concurso de fondos para la investigación que dispone Capic para el fomento y desarrollo de la investigación en Contabilidad y áreas afines.

2 Académico Facultad de Economía y Negocios, Universidad de Chile (Chile), ljaras@fen.uchile.cl.

3 Académico Facultad de Ciencias Económicas y Administrativas, Universidad de Concepción (Chile), benvenuto@udec.cl

4 Académico Facultad de Ciencias Económicas y Jurídicas, Universidad de Magallanes (Chile), ricardo.mendez@umag.cl 


\begin{abstract}
Currently, support for university scientific production focuses on networking creation. In the same spirit, the year 1990, Scientific Society CAPIC, Permanent Academic Conference on Research Accounting, was created. This institution houses primarily academics attached to Chilean universities, both public and private. In this context, the purpose of this research is related to identify to what extent the participation of investigators in CAPIC, during the period 1992-2015 has generated collaborative networks, and also to identify the main attributes and internal structures of such networks. The findings clearly demonstrate that the macro network generated during the last two decades has low cohesion with high fragmentation, and a prevalence of the male gender. From a micro perspective, the analysis of the eight main networks, evidences a prevalence of certain universities, being the micro network of Pontificia Universidad Católica de Valparaíso the one with a greater number of participants. Furthermore, it is observed that relevant positions concerning connection and closeness are being assumed by women; even when the male gender predominates in most networks. Finally, another result is associated to the existence of a micro network internationally connected, in particular with researchers from Colombia, Argentina y Chile. Future research should focus on the identification of the networks capitals with the aim to display and manage such resources -economic, intellectual, cultural and social resources- to promote connectivity between researchers with common interests, facilitating the localization of networks with structural advantages that promote a higher productivity among the academics participating in CAPIC.
\end{abstract}

Keywords: Networks analysis, CAPIC, co-authorship, research networks.

\section{INTRODUCCIÓN}

Los académicos universitarios representan uno de los pilares fundamentales en la generación de conocimiento, en esta línea, diversas universidades nacionales han generado planes de incentivo para aumentar la producción científica, principalmente orientada a la publicación en revistas de prestigio internacional. En este sentido, uno de los métodos de fomento a la producción académica se sustenta en el trabajo colaborativo, en especial el interdisciplinario.

Por consiguiente, la generación de redes académicas o redes de conocimiento se ha transformado en una de las principales vías para fomentar el aumento en las contribuciones intelectuales.

Es así como las redes de conocimiento, según Prada (2005, citado en Cárdenas, 2016) persiguen propósitos, tales como: mejorar la calidad del trabajo académico y científico; optimizar la gestión del conocimiento; crear y fortalecer el aprovechamiento de recursos, y posibilitar el flujo de información; así también: producir, almacenar y distribuir conocimiento científico por medio de cualquier método de transmisión tecnológica. Lo anterior, con el objeto de transformar el entorno en la búsqueda constante del enriquecimiento intelectual.
Por su lado, para Cárdenas (2016) la cohesión de una red de conocimiento se afecta, significativamente, por el número de miembros; por el nivel de competencia; por la existencia de relaciones estables; así como por el tipo de metas definidas para el grupo colaborativo.

El producto de las colaboraciones académicas debe ser socializado entre la comunidad científica. En este sentido, los encuentros académicos -congresos, conferencias, simposios, por mencionar algunas categorías- fomentan el intercambio de ideas y contribuyen a la generación de redes de colaboración entre investigadores. En esta línea, y en particular en nuestro entorno, existen encuentros académicos orientados a las Ciencias Económicas y Empresariales, particularmente en el campo de la Contabilidad y disciplinas afines se creó, en el año de 1990, la Conferencia Académica Permanente de Investigación Contable-Capic-. El propósito principal de esta sociedad científica es fomentar la producción intelectual de académicos pertenecientes a universidades nacionales que dictan la carrera de Contador Público y/o Auditor. En este sentido, a partir de 1991 comenzaron las reuniones anuales. Para fines de la década del 2000 existían más de veinte universidades chilenas, tanto públicas como privadas, adscritas a la Capic (González, 2008). 
En cuanto al análisis de las redes de conocimiento, Wasserman y Faust (2009) indican que existen diversos métodos para comprender la colaboración científica, entre ellas se encuentran los cuestionarios, las entrevistas, las observaciones, las bases de datos, y las técnicas sociométricas. En este aspecto, uno de los métodos más utilizado para identificar y comprender las redes de colaboración científica o académicas corresponde a los análisis de coautoría en publicaciones (Lu y Feng, 2008).

Por todo lo anterior, surgen diversas interrogantes en relación a la generación de conocimiento en torno a la Capic, particularmente en función a cómo se ha desarrollado el trabajo colaborativo entre los académicos. Es así como entre las preguntas de investigación que guiaron el desarrollo de este trabajo, están: ¿cuál es la proporción de trabajos colaborativos?, ¿cuál es el número de investigadores que realizaron artículos en coautoría?, ¿se ha generado una red de colaboración cohesionada?, o ¿existe fragmentación de la red?, ¿qué características poseen las micro redes que se desprenden de la red generada por la Capic?, y por último, ¿qué relación de género se presentó en las redes configuradas en torno a la Conferencia?

Con el propósito de dar respuesta a las interrogantes antes expuestas se ha definido como objetivo de investigación el identificar en qué medida la participación de investigadores en la Capic, durante el período 1993-2015, ha generado redes de colaboración. Por otro lado, se pretende conocer los atributos principales, así como las estructuras internas de dichas redes que se conformaron en base a las coautoría de los artículos académicos.

Luego de esta introducción se expone la estrategia adoptada en función de los criterios metodológicos que permitieron desarrollar el estudio, posteriormente se presentan los principales resultados, para enseguida, dar paso a la conclusiones, recomendaciones e implicancias futuras de esta investigación.

\section{MÉTODO, MATERIALES Y METODOLOGÍA}

Para alcanzar el objetivo ante descrito, se utilizó, en la presente investigación, un enfoque cuantitativo de tipo exploratorio y de alcance longitudinal. El objeto de análisis se centró en las relaciones de colaboración académica que se desprenden de las coautorías de artículos aceptados por el Comité Técnico de Capic para ser presentados en las conferencias organizadas por la sociedad científica durante los años 1992 a 2015. Se consideraron solo los trabajos que contaron con evaluación del Comité Técnico constituido para cada año, de este modo, se descartaron los artículos presentados bajo otra modalidad.

En cuanto a la recolección de los artículos académicos, Capic no dispone de un repositorio -ni físico, ni virtual-. Por tal motivo, se procedió a contactar a diversos académicos que participaron en la organización de algunas de las veinticuatro versiones de la conferencia bajo estudio. Es así, como se pudo acceder, por un lado, a los programas académicos; mientras que por otro, a los artículos académicos que fueron aceptados por el Comité Técnico evaluador. En este último caso, para algunos períodos se recolectaron artículos en formato impreso, y en otros, en formato PDF o Word.

Sin embargo, no se pudo contar con información alguna para cuatro períodos. En concreto, nos referimos a las conferencias de los años: 1992, 1995, 2000, y 2003.

En definitiva, se accedió, ya sea por medio del programa académico como de los propios artículos, a información relacionada con 246 trabajos académicos. La distribución por período se puede observar en la Tabla 1, así como los datos relacionados con el itinerario de la conferencia durante el período bajo análisis. 
Tabla 1. Itinerario y número de artículos aceptados por Comité Técnico de la Capic (1992-2015).

\begin{tabular}{llccc}
\hline Período & \multicolumn{1}{c}{ Institución sede } & Ciudad & Zona & $\begin{array}{c}\text { Número } \\
\text { de artículos }\end{array}$ \\
\hline 1992 & Pontificia Universidad Católica de Valparáso & Valparaíso & Norte & ND \\
1993 & Universidad Austral de Chile & Puerto Montt & Sur & 11 \\
1994 & Universidad de Valparaíso & Valparaíso & Norte & 9 \\
1995 & Universidad de Los Lagos & Osorno & Sur & ND \\
1996 & Universidad de La Serena & La Serena & Norte & 8 \\
1997 & Universidad Católica del Norte & Antofagasta & Norte & 12 \\
1998 & Universidad de Concepción & Concepción & Sur & 6 \\
1999 & Universidad Arturo Prat & Iquique & Norte & 11 \\
2000 & Universidad de Talca & Talca & Sur & ND \\
2001 & Universidad de Tarapacá & Arica & Norte & 7 \\
2002 & Universidad de Magallanes & Punta Arenas & Sur & 4 \\
2003 & Universidad Diego Portales & Santiago & Centro & ND \\
2004 & Universidad de La Frontera & Temuco & Sur & 10 \\
2005 & Universidad Católica de la Santísima Concepción & Concepción & Sur & 12 \\
2006 & Universidad del Bio Bio & Chillán & Sur & 9 \\
2007 & Universidad de La Serena & La Serena & Norte & 13 \\
2008 & Pontificia Universidad Católica de Valparaíso & Valparaíso & Norte & 9 \\
2009 & Universidad Arturo Prat & Iquique & Norte & 26 \\
2010 & Universidad de Chile & Santiago & Centro & 15 \\
2011 & Universidad Católica del Norte & Antofagasta & Norte & 17 \\
2012 & Universidad de Magallanes & Punta Arenas & Sur & 15 \\
2013 & Universidad Austral de Chile & Valdivia & Sur & 17 \\
2014 & Universidad de La Frontera & Temuco & Sur & 12 \\
2015 & Pontificia Universidad Católica de Valparáso & Valparaíso & Norte & 23 \\
\hline & Total artículos & & 246 \\
\hline \hline
\end{tabular}

ND: No Disponible.

Fuente: elaboración propia.

Una vez recolectados los antecedentes -programas académicos de las conferencias y/o artículos de investigación-, se procedió a identificar el número de artículos que fueron elaborados bajo la modalidad de coautoría, es decir: en la confección de la investigación participaron más de un investigador. En tal sentido, se puede observar en la Tabla 2, como un 33,7\% de los 246 artículos fueron elaborados por un solo investigador. Por otro lado, en dicha Tabla se aprecia como las coautoría entre dos investigadores predominan con un $42,7 \%$ del total. 
Tabla 2. Distribución del número de artículos académicos presentados a la Capic, según el número de autores.

\begin{tabular}{|c|c|c|c|c|c|c|c|}
\hline \multirow{2}{*}{ Año } & \multirow{2}{*}{$\begin{array}{c}\text { Total } \\
\text { artículos }(\mathrm{N})\end{array}$} & \multicolumn{6}{|c|}{ Distribución por número de autores } \\
\hline & & uno & dos & tres & cuatro & cinco & seis \\
\hline 1993 & 11 & 6 & 4 & 1 & 0 & 0 & 0 \\
\hline 1994 & 9 & 6 & 2 & 0 & 0 & 0 & 1 \\
\hline 1996 & 8 & 2 & 5 & 1 & 0 & 0 & 0 \\
\hline 1997 & 12 & 9 & 3 & 0 & 0 & 0 & 0 \\
\hline 1998 & 6 & 1 & 2 & 3 & 0 & 0 & 0 \\
\hline 1999 & 11 & 6 & 3 & 1 & 0 & 0 & 1 \\
\hline 2001 & 7 & 3 & 4 & 0 & 0 & 0 & 0 \\
\hline 2002 & 4 & 2 & 2 & 0 & 0 & 0 & 0 \\
\hline 2004 & 10 & 2 & 5 & 2 & 1 & 0 & 0 \\
\hline 2005 & 12 & 6 & 5 & 0 & 1 & 0 & 0 \\
\hline 2006 & 9 & 2 & 6 & 1 & 0 & 0 & 0 \\
\hline 2007 & 13 & 4 & 8 & 0 & 1 & 0 & 0 \\
\hline 2008 & 9 & 3 & 4 & 1 & 1 & 0 & 0 \\
\hline 2009 & 26 & 7 & 11 & 6 & 1 & 1 & 0 \\
\hline 2010 & 15 & 5 & 5 & 4 & 0 & 1 & 0 \\
\hline 2011 & 17 & 4 & 4 & 6 & 3 & 0 & 0 \\
\hline 2012 & 15 & 5 & 6 & 1 & 3 & 0 & 0 \\
\hline 2013 & 17 & 5 & 6 & 4 & 1 & 0 & 1 \\
\hline 2014 & 12 & 1 & 9 & 1 & 1 & 0 & 0 \\
\hline 2015 & 23 & 4 & 11 & 5 & 3 & 0 & 0 \\
\hline Total & 246 & 83 & 105 & 37 & 16 & 2 & 3 \\
\hline Proporció & obre N & $33,7 \%$ & $42,7 \%$ & $15,0 \%$ & $6,5 \%$ & $0,8 \%$ & $1,2 \%$ \\
\hline Proporció1 & cumulada & $33,7 \%$ & $76,4 \%$ & $91,5 \%$ & $98,0 \%$ & $98,8 \%$ & $100,0 \%$ \\
\hline
\end{tabular}

Fuente: elaboración propia.

En consecuencia, los artículos académicos confeccionados bajo la modalidad de coautoría ascendieron a: 163 , esto es $66,26 \%$ del total de artículos aprobados por el Comité Técnico de la Capic durante los veinte años a los cuales se obtuvo acceso a información.

Posteriormente, con el propósito de identificar el número de investigadores que se relacionan con los 163 artículos académicos elaborados bajo la modalidad de coautoría, se confeccionó una base de datos relacional. Para lo anterior, en primer lugar, se generó un archivo de texto en formato txt, mediante pares de autores que se identificaron en las coautorías de los artículos de investigación. Posteriormente, a través del uso de los programas informáticos: Ucinet y NetDraw, se procedió a convertir el archivo txt a una base de datos relacional direccionada. Como resultado de lo anterior, se identificó a 217 investigadores que colaboraron en la modalidad de coautorías. No obstante, las relaciones de coautoría son definidas como no dirigidas (Wasserman y Faust, 2009), esto debido a que en la confección de un artículo académico en coautoría, los investigadores se relacionan entre ellos. Así por ejemplo: el investigador_1 trabaja colaborativamente con el investigador_2 y viceversa. Para ilustrar mejor, en la Tabla 3 se puede apreciar una matriz relacional no dirigida-simétrica-, en donde cuatro investigadores, combinados entre ellos, 
producen dos artículos bajo coautoría, esto es: por un lado, un artículo entre el investigador_1, el investigador_2, el investigador_3, y el investigador_4; mientras que por otro, un artículo bajo coautoría entre el investigador_3 y el investigador_4. Los valores asignados en la matriz corres- ponden a: cero (0) cuando no existe colaboración de coautoría entre los investigadores; y uno (1) cuando si existe colaboración de coautoría. De forma adicional, en la mencionada Tabla se observa la representación gráfica que se obtiene de la matriz relacional a través del uso de grafos.

Tabla 3. Ejemplo de matriz relacional no dirigida (simétrica) y su respectiva representación gráfica a través de grafo.

Investigador_1 Investigador_2 Investigador_3 Investigador_4

Grafo

\begin{tabular}{l|c|c|c|c|c|}
\hline Investigador_1 & 0 & 1 & 1 & 1 \\
\cline { 2 - 5 } & 1 & 0 & 0 & 0 \\
\hline Investigador_2 & 1 & 0 & 0 & 0 \\
\cline { 2 - 5 } & 1 & 0 & 1 & 1 \\
\hline
\end{tabular}

Fuente: elaboración propia en base a programas informáticos: Ucinet y NetDraw.

Por otro lado, se generó una matriz asimétrica en función a diversas características o atributos de los 217 investigadores involucrados en la elaboración de los 163 artículos bajo coautoría. En esta línea, se definieron los siguientes atributos: a) género del investigador; b) afiliación universidad; c) país de la universidad; d) número de años con trabajos colaborativos o de coautoría. Por otra parte, el período de tiempo bajo análisis se dividió en tres intervalos: el primero va desde 1993 a 2002; el segundo desde 2004 a 2010; mientras que el último, se relaciona con los años 2011 a 2015. Con estos tres intervalos se crearon dos nuevos atributos para cada investigador. El primero se relaciona al intervalo al cual pertenece el primer trabajo colaborativo elaborado por cada autor; mientras que el segundo, se vincula con el intervalo al que corresponde el último trabajo de coautoría realizado. Con estas dos últimas caracterizaciones se persigue observar el estado de la red a la cual pertenece un investigador, es decir: activa, desactiva; así como la antigüedad o vigencia de la misma.

En cuanto a las técnicas de análisis, se utilizó el Análisis de Redes Sociales (Wasserman y Faust, 2009; Gaete y Vásquez 2008; Olmeda, Perianes y Ovalle, 2008; Sanz, 2003). En particular, se utilizaron técnicas estadísticas relacionadas con: cohesión y centralidad de la red (Cárdenas,
2016). A su vez, para representar las relaciones de coautoría, así como la configuración de redes, se utilizó la técnica de grafos (Cárdenas, 2016). Los programas informáticos utilizados fueron: Ucinet 6 para el análisis de redes sociales (Borgatti, Everett y Freeman, 2002), y NetDraw para la representación gráfica de las redes a través de grafos (Borgatti, 2002).

\section{RESULTADOS}

En relación con la estructura general de la red sociométrica de coautoría generada durante los veinte años de análisis de la Capic, la Tabla 4 nos proporciona sus principales propiedades, así como un diagrama mediante grafos. En este sentido, de los 217 investigadores un 36\% son mujeres, por otro lado, uno de los aspectos más importante se centra en la fragmentación de la red, la cual posee un valor de 0,9624-Fragmentation-. Esto se materializa en la existencia de diversos componentes o micro redes al interior de la macro red conformada por los 217 investigadores. En concreto, se observa la existencia de 46 componentes -Components-. Otras propiedades de interés se relacionan con una baja densidad, esto es: 0,0122-Density-, lo cual se refiere a una baja interacción entre los investigadores; 
en promedio cada investigador se relacionó con casi tres colegas, esto es: 2,6267-Avg. Degree-, no obstante, la dispersión es alta - Std. Dev. Degree-, llegando a casi a dos investigadores (1,7317). Por otro lado, al menos un investigador solo realizó trabajos colaborativos con un investigador $-\mathrm{Mi}$ nimun Degree-; mientras que por otro, al menos un investigador se relacionó con once colegas diferente-Maximun Degree-. Por último, un indicador interesante de analizar lo constituye el diámetro de la red-Diameter-, para este caso, su valor corresponde a seis. Lo anterior, significa que el recorrido máximo de la información y recursos compartido entre dos investigadores localizados en puntos extremos del componente o micro red es a través de la intervención de seis académicos, es decir, esta situación afecta la cohesión de la red, mientras menor es la distancia mayor es la cohesión.

Tabla 4. Propiedades y diagrama de la red sociométrica (macro red) generada por la colaboración académica en la Capic (1993-2015).

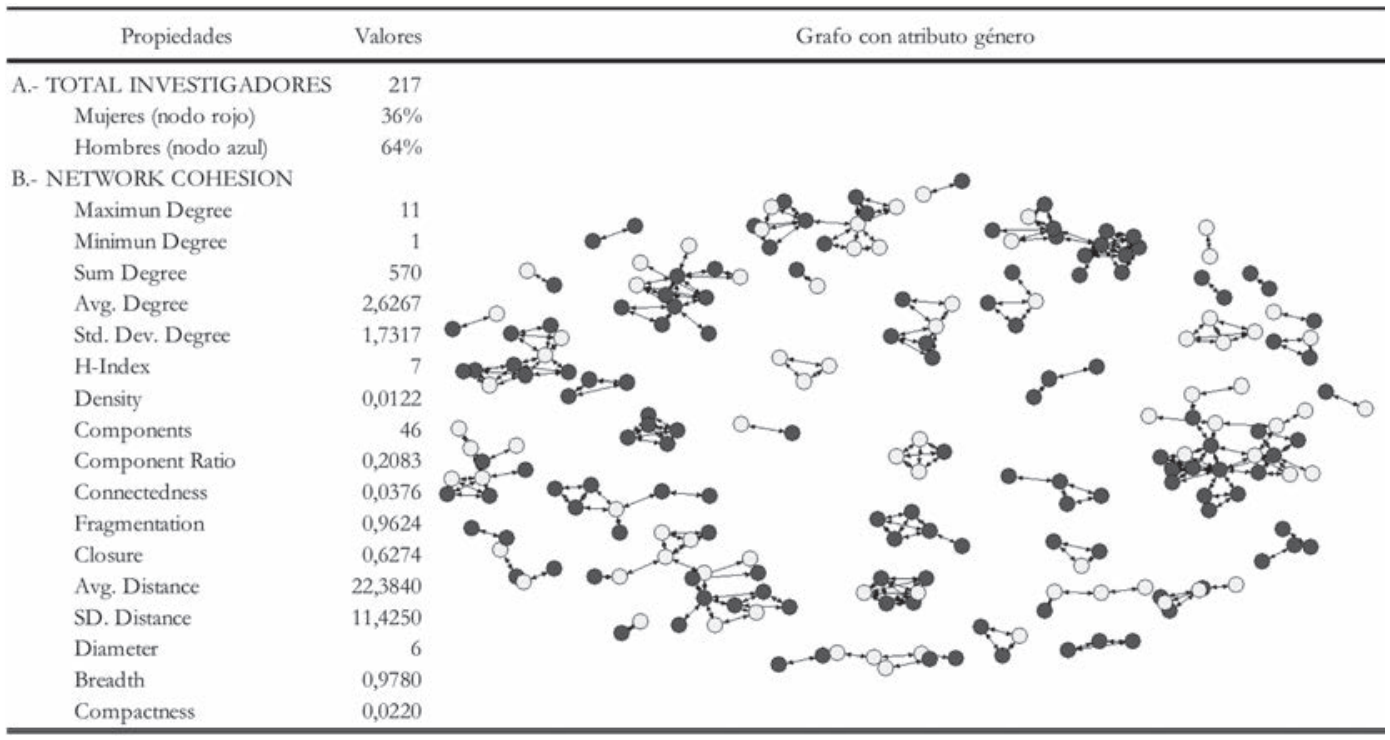

Fuente: elaboración propia en base a programas informáticos: Ucinet y NetDraw.

Como se afirmó antes, la red compuesta por los 217 investigadores presenta una alta fragmentación, identificándose 46 componentes o micro redes. En este aspecto, la Tabla 5. proporciona información relacionada con la composición de dichas micro redes. En ella se observa que la micro red con mayor número de miembros tiene 24 investigadores; seguida por una de 17 actores. En el otro extremo, se aprecia que colaboraciones en torno a dos investigadores se presentaron en 19 micro redes. 
Tabla 5. Descripción de los componentes (micro redes) que conforman la macro red de colaboración académica en la Capic (1993-2015)

\begin{tabular}{ccccc}
\hline $\begin{array}{c}\text { Número de } \\
\text { componentes } \\
\text { (micro redes) }\end{array}$ & Tamaño & $\begin{array}{c}\text { Total } \\
\text { investigadores }\end{array}$ & Proporción & $\begin{array}{c}\text { Proporción } \\
\text { acumulada }\end{array}$ \\
\hline 1 & 24 & 24 & $11,06 \%$ & $11,06 \%$ \\
1 & 17 & 17 & $7,83 \%$ & $18,89 \%$ \\
1 & 14 & 14 & $6,45 \%$ & $25,35 \%$ \\
1 & 13 & 13 & $5,99 \%$ & $31,34 \%$ \\
1 & 12 & 12 & $5,53 \%$ & $36,87 \%$ \\
1 & 10 & 10 & $4,61 \%$ & $41,47 \%$ \\
1 & 9 & 9 & $4,15 \%$ & $45,62 \%$ \\
1 & 7 & 7 & $3,23 \%$ & $48,85 \%$ \\
2 & 6 & 12 & $5,53 \%$ & $54,38 \%$ \\
2 & 5 & 10 & $4,61 \%$ & $58,99 \%$ \\
6 & 4 & 24 & $11,06 \%$ & $70,05 \%$ \\
9 & 3 & 27 & $12,44 \%$ & $82,49 \%$ \\
19 & 2 & 38 & $17,51 \%$ & $100,00 \%$ \\
\hline 46 & & 217 & & \\
\hline \hline
\end{tabular}

Fuente: elaboración propia en base a programa informático: Ucinet.

A continuación nos centraremos en el análisis de las ocho principales micro redes, en función al número de investigadores que la conforman. En este aspecto, la Figura 1 nos muestra, a través de grafos, las representaciones de las ocho principales micro redes, mientras que por otro lado, la Tabla 6 nos proporciona antecedentes relacionados con los atributos de los investigadores que conforman cada micro red.

La principal red -Micro red 1- quedó conformada por 24 investigadores, donde el 41,67\% son mujeres; el $100 \%$ de sus miembros trabaja en universidades nacionales. En este sentido, el 79,17\% está adscrito a la Pontificia Universidad Católica de Valparaíso -PUCV-; el 16,67\% a la Universidad de Chile-UdeCh-; mientras el $4,17 \%$ corresponde a una investigadora que en sus inicios estaba vinculada a la PUCV, pero posteriormente se trasladó a la UdeCh. En relación a la colaboración entre investigadores de la micro red, se observa que el 58,33\% realizó su último trabajo de colaboración académica entre los años 2011-2015; mientras que el 45,83\% realizó su primera contribución a la Capic entre los años 19932002. Por último, el 37,50\% de los investigadores de la Micro red 1 han participado solo en un año con trabajos en coautoría; mientras que un 4,17\% lo ha realizado durante nueve de los veinte años bajo análisis.

Siguiendo el análisis con la segunda micro red, podemos observar que su composición está basada en 17 investigadores, donde el 47,06\% son mujeres; el $100 \%$ de sus miembros son de universidades nacionales. Para este caso: el $64,71 \%$ se vincula a la Universidad Católica del Norte-UCN-; $29,41 \%$ a la Universidad de Santiago de Chile-USACh-; mientras el 5,88\%, corresponde a una investigadora que en sus inicios estaba adscrita a la UCN, pero posteriormente se cambió a la USACh. En relación a la colaboración entre investigadores de la micro red, se observa que el $76,47 \%$ realizó su último trabajo bajo colaboración académica entre los años 2011-2015; mientras que el 58,82\% desarrolló su primera contribución a la Capic entre los años 2011-2015. Para finalizar el análisis a la Micro red 2 , podemos señalar que el $58,82 \%$ de los investigadores han participado con artículos en coautoría solo en un año; mientras que un 5,88\% lo ha realizado durante cuatro años.

En cuanto a los atributos de la Micro red 3, se observa que la conforman 14 investigadores, 
donde el 14,15\% son mujeres; el $100 \%$ de sus miembros pertenece a universidades nacionales. Es decir: el 92,86\% pertenece a la Universidad Austral de Chile-UACh-; mientras que el $7,14 \%$ a la Universidad de La Frontera -UFRO-. Por otro lado, en relación a la colaboración entre investigadores de la micro red, se observa que el 78,57\% desarrolló su último trabajo bajo colaboración académica entre los años 2011-2015; mientras que el $50 \%$ realizó su primera contribución a la Capic entre los años 2011-2015. Para finalizar el análisis a la Micro red 3, señalar que el $71,43 \% \%$ de los investigadores han participado solo en un año en la modalidad de coautoría; mientras que un $7,18 \%$ lo ha realizado durante seis años.

Por su lado, la Micro red 4 quedó constituida por 13 investigadores, de ellos, el 46,15\% son mujeres; el $100 \%$ de sus miembros pertenece a universidades nacionales. En concreto, el 46,15\% se asocia a la Universidad Diego Portales -UDP; el mismo porcentaje anterior a la Universidad de Chile-UdeCh-; y por último, el 7,69\% corresponde a una investigadora que en sus inicios estaba adscrita a la UDP, pero posteriormente se trasladó a la Universidad Finis Terrae -UFT-. Por otro lado, en relación a la colaboración entre investigadores de la micro red, se observa que el $69,23 \%$ realizó su último trabajo bajo colaboración académica entre los años 2011-2015; mientras que el $53,85 \%$ desarrolló su primera contribución a la Capic entre los años 2004-2010. A su vez, en relación a la proporción de investigadores, así como a la cantidad de años de trabajo colaborativo, se aprecia que el 76,92\% de los investigadores solo han participado en un año de la conferencia; mientras que el resto lo ha hecho en tres años.

Por su lado, la Micro red 5 se constituyó por 12 investigadores, de ellos, el 33,33\% son mujeres; el $100 \%$ de sus miembros pertenece a universidades nacionales. Esto es el $66,67 \%$ se asocia a la Universidad de Magallanes -UMAG-; el $25,00 \%$ a la Universidad Austral de Chile -UACh-; y por último, el 8,33\% corresponde a un investigador que en sus inicios estaba adscrito a la UACh, y posteriormente se trasladó a la UMAG. Por otro lado, en relación a la colaboración entre investigadores de la micro red, se observa que el 75,00\% de ellos realizó su último trabajo bajo colaboración académica entre los años 2004-2010; mientras que el 66,67\% desarrolló su primera contribución a la Capic en el mismo intervalo de tiempo antes mencionado. Por su lado, en relación a la proporción de investigadores y la cantidad de años en los cuales trabajaron colaborativamente, se observa que el $75,00 \%$ solo ha participado en un año de la Conferencia; mientras que el 8,33\% ha participado en tres años.

En relación a los atributos de la Micro red 6, podemos indicar que está constituida por 10 investigadores, de ellos, el 30,00\% son mujeres; el $100 \%$ de sus miembros pertenece a universidades nacionales. Es decir el $60,00 \%$ se adscribe a la Universidad Católica de la Santísima Concepción-UCSC-; el 30,00\% a la Universidad de Las Américas -UDLA-; y por último, el 10,00\% corresponde a una movilidad, en este caso se trata de una investigadora inicialmente adscrita a la UCSC, que posteriormente se trasladó a la UDLA. Por su parte, en relación a la colaboración entre investigadores de la micro red, se observa que el 30,00\% de ellos realizó su último trabajo, bajo colaboración académica, entre los años 2010-2015; mientras que el 90,00\% desarrolló su primera contribución a la Capic en el intervalo de tiempo que transcurre entre 20042010. Por último, se observa que un $30 \%$ de los investigadores solo ha participado, de forma colaborativa, durante un año, mientras que el 20\% de ellos lo ha hecho en cuatro años.

En cuanto a los atributos de la Micro red 7, se observa que la conforman 9 investigadores, donde el 55,56\% son mujeres; es la única red en la cual existe presencia de investigadores extranjeros. Concretamente el 55,56\% proviene de Colombia; el 22,22\% de Argentina; e igual porcentaje de Chile. En relación a las universidades de procedencia, se aprecia que un $55,56 \%$ de los investigadores está asociado a la Universidad Santo Tomás de Colombia -UST (Col.) -; por otro lado, el 22,22\% pertenece a la Universidad Nacional del Litoral de Argentina -UNL (Arg)-; mientras, por último, el 22,22\% está adscrito a la Universidad de Santiago de Chile -USACh-. Asimismo, en relación a la colaboración entre investigadores de la micro red, se observa que el 77,78\% desarrolló su último trabajo colaborativo entre los años 2011-2015; y por otro lado, el 90,00\% realizó su primera contribución a la Capic entre los años 2004-2010. Para finalizar el análisis a la Micro red 7, señalar que el 66,67\% de los investigadores han participado, de forma colaborativa, solo en un año; mientras que un 22,22\% lo ha realizado durante cinco años.

Para finalizar el análisis a los atributos de los investigadores pertenecientes a las micro redes, podemos observar que la Micro red 8 quedó conformada por 7 investigadores, de ellos el 14,29\% son mujeres; el $100 \%$ de sus miembros son de universidades nacionales. En concreto el 85,71\% 
se asocia a la Universidad Nacional Andrés Be1lo-UNAB-; mientras que el resto a la Universidad Tecnológica Metropolitana-UTEM-. Por su lado, en relación a la colaboración entre investigadores de la micro red, se observa que el $57,14 \%$ realizó su último trabajo bajo colaboración académica entre los años 2011-2015; mientras que el $85,71 \%$ desarrolló su primera contribución a la Capic entre los años 2004-2010. En cuanto al número de años en los cuales han tenido presencia con trabajos colaborativos, el $42,86 \%$ de los investigadores pertenecientes a esta micro red solo presentó trabajos para un año; mientras que el 28,57\% de los investigadores ha participado colaborativamente durante cuatro años.

En consecuencia a todo lo expuesto anteriormente, proponemos diversas clasificaciones para las ocho principales micro redes de colaboración académica bajo análisis, en concreto:

- En cuanto al género:

- Redes de alto predominio masculino $(>65 \%)$ : Micro red 3; Micro red 8; Micro red 6; y Micro red 5.

- Redes de predominio masculino (>50\% $y<66 \%$ ): Micro red 1; Micro red 4; y Micro red 2.

- Redes de predominio femenino (>50\% y $<66 \%$ ): Micro red 7 .

- En cuanto a la internacionalización:

- Red internacional: Micro red 7.

- Redes netamente nacionales: Micro red 1; Micro red 2; Micro red 3; Micro red 4; Micro red 5; Micro red 6; y Micro red 8 .
- En cuanto a la movilidad interuniversitaria de sus miembros:

- Redes con movilidad: Micro red 1; Micro red 2; Micro red 4; Micro red 5; y Micro red 6

- Redes sin movilidad: Micro red 3; Micro red 7; y Micro red 8.

- En cuanto a su estado:

- Redes activas (>50\% última coautoría en intervalo 2010-2015): Micro red 3; Micro red 7; Micro red 2; Micro red 4; y Micro red 1.

- Redes parcialmente activas (>50\% última coautoría en intervalo 2004-2010 y presencia en intervalo 2011-2015): Micro red 6.

- Redes inactivas (sin presencia de coautoría en intervalo 2011-2015): Micro red 5.

- En cuanto a la consolidación:

- Redes altamente consolidadas (entre sus miembros, existe colaboración durante más de siete años): Micro red 1.

- Redes consolidadas (entre sus miembros, existe colaboración durante más de tres y menos de siete años): Micro red 3; Micro red 7; Micro red 2; y Micro red 6.

- Redes en vías de consolidación (entre sus miembros, existe colaboración durante menos de cuatro años): Micro red $4 \mathrm{y}$ Micro red 5. 
Figura 1. Estructuras de las ocho principales micro redes de colaboración académica en la Capic (1993-2015).

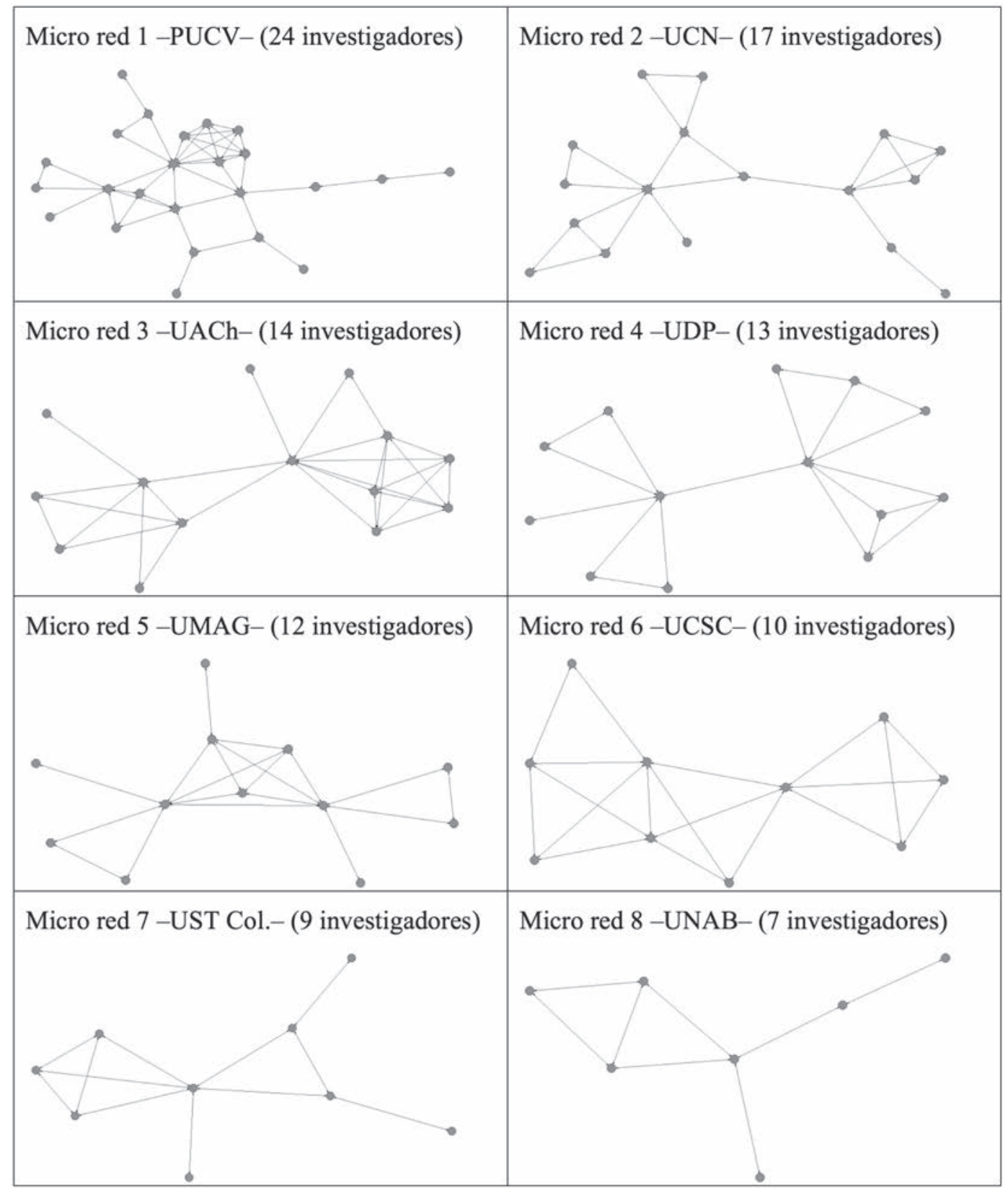

Fuente: elaboración propia en base a programa informático: NetDraw. 
Tabla 6. Atributos de las ocho principales micro redes de colaboración académica en la Capic (1993-2015).

\begin{tabular}{|c|c|c|c|c|c|c|c|c|}
\hline \multirow{3}{*}{ Atributos } & \multicolumn{8}{|c|}{ Denominación $\mathrm{y}$ valores de las principales micro redes } \\
\hline & Micro red 1 & Micro red 2 & Micro red 3 & Mero red 4 & Micro red 5 & Micro red 6 & Micro red 7 & Micro red 8 \\
\hline & PUCV & UCN & $\mathrm{UACh}$ & UDP & UMAG & UCSC & $\overline{\text { UST (COL) }}$ & UNAB \\
\hline A. TOTAL INVESTIGADORES & 24 & 17 & 14 & 13 & 12 & 10 & 9 & 7 \\
\hline \multicolumn{9}{|l|}{ B.- RELACIÓN GÉNERO } \\
\hline Mujeres & $41,67 \%$ & $47,06 \%$ & $14,29 \%$ & $46,15 \%$ & $33,33 \%$ & $30,00 \%$ & $55,56 \%$ & $14,29 \%$ \\
\hline Hombres & $58,33 \%$ & $52,94 \%$ & $85,71 \%$ & $53,85 \%$ & $66,67 \%$ & $70,00 \%$ & $44,44 \%$ & $85,71 \%$ \\
\hline \multicolumn{9}{|l|}{ C. RELACIÓN PAISS } \\
\hline Chile & $100,00 \%$ & $100,00 \%$ & $100,00 \%$ & $100,00 \%$ & $100,00 \%$ & $100,00 \%$ & $22,22 \%$ & $100,00 \%$ \\
\hline Colombia & & & & & & & $55,56 \%$ & \\
\hline Argentina & & & & & & & $22,22 \%$ & \\
\hline \multicolumn{9}{|l|}{ D.- RELACIÓN UNIVERSIDADES } \\
\hline PUCV & $79,17 \%$ & & & & & & & \\
\hline $\mathrm{PUCV} \rightarrow \mathrm{UdeCh}$ & $4,17 \%$ & & & & & & & \\
\hline UdeCh & $16,67 \%$ & & & $46,15 \%$ & & & & \\
\hline UCN & & $64,71 \%$ & & & & & & \\
\hline $\mathrm{UCN} \rightarrow \mathrm{USACh}$ & & $5,88 \%$ & & & & & & \\
\hline USACh & & $29,41 \%$ & & & & & $22,22 \%$ & \\
\hline $\mathrm{UACh}$ & & & $92,86 \%$ & & $25,00 \%$ & & & \\
\hline UFRO & & & $7,14 \%$ & & & & & \\
\hline UDP & & & & $46,15 \%$ & & & & \\
\hline $\mathrm{UDP} \rightarrow \mathrm{UFT}$ & & & & $7,69 \%$ & & & & \\
\hline UACh $\rightarrow$ UMAAG & & & & & $8,33 \%$ & & & \\
\hline UMAG & & & & & $66,67 \%$ & & & \\
\hline UCsC & & & & & & $60,00 \%$ & & \\
\hline $\mathrm{UCSC} \rightarrow$ UDLA & & & & & & $10,00 \%$ & & \\
\hline UDLA & & & & & & $30,00 \%$ & & \\
\hline UST (COL) & & & & & & & $55,56 \%$ & \\
\hline UNL (ARG) & & & & & & & $22,22 \%$ & \\
\hline UNAB & & & & & & & & $85,71 \%$ \\
\hline UTEM & & & & & & & & $14,29 \%$ \\
\hline \multirow{2}{*}{\multicolumn{9}{|c|}{$\begin{array}{l}\text { E. RELACIÓN PERIODO PRIMERA } \\
\text { COAUTORLA }\end{array}$}} \\
\hline & & & & & & & & \\
\hline 1993-2002 & $45,83 \%$ & $41,18 \%$ & $14,29 \%$ & & $33,33 \%$ & & $22,22 \%$ & \\
\hline $2004-2010$ & $20,83 \%$ & & $35,71 \%$ & $53,85 \%$ & $66,67 \%$ & $90,00 \%$ & $22,22 \%$ & $85,71 \%$ \\
\hline 2011-2015 & $33,33 \%$ & $58,82 \%$ & $50,00 \%$ & $46,15 \%$ & & $10,00 \%$ & $55,56 \%$ & $14,29 \%$ \\
\hline F. RELACIÓN PERIODO ÚLTIM & & & & & & & & \\
\hline \multicolumn{9}{|l|}{ COAUTORLA } \\
\hline 1993-2002 & $16,67 \%$ & $23,53 \%$ & & & $25,00 \%$ & & $11,11 \%$ & \\
\hline $2004-2010$ & $25,00 \%$ & & $21,43 \%$ & $30,77 \%$ & $75,00 \%$ & $70,00 \%$ & $11,11 \%$ & $42,86 \%$ \\
\hline 2011-2015 & $58,33 \%$ & $76,47 \%$ & $78,57 \%$ & $69,23 \%$ & & $30,00 \%$ & $77,78 \%$ & $57,14 \%$ \\
\hline \multirow{2}{*}{\multicolumn{9}{|c|}{$\begin{array}{l}\text { G. RELACIÓN AÑOS DE TRABAJO } \\
\text { COLABORATIVO }\end{array}$}} \\
\hline & & & & & & & & \\
\hline Un año & $37,50 \%$ & $58,82 \%$ & $71,43 \%$ & $76,92 \%$ & $75,00 \%$ & $30,00 \%$ & $66,67 \%$ & $42,86 \%$ \\
\hline Dos años & $25,00 \%$ & $11,76 \%$ & & & $16,67 \%$ & $30,00 \%$ & & $14,29 \%$ \\
\hline Tres años & $8,33 \%$ & $23,53 \%$ & $14,29 \%$ & $23,08 \%$ & $8,33 \%$ & $20,00 \%$ & $11,11 \%$ & $14,29 \%$ \\
\hline Cuatro años & $12,50 \%$ & $5,88 \%$ & & & & $20,00 \%$ & & $28,57 \%$ \\
\hline Cinco años & $4,17 \%$ & & $7,14 \%$ & & & & $22,22 \%$ & \\
\hline Seis años & $4,17 \%$ & & $7,14 \%$ & & & & & \\
\hline \multicolumn{9}{|l|}{ Siete años } \\
\hline Ocho años & $4,17 \%$ & & & & & & & \\
\hline Nueve años & $4,17 \%$ & & & & & & & \\
\hline
\end{tabular}

Fuente: elaboración propia en base a programa informático: Ucinet.

Acerca de las propiedades estructurales de las micro redes generadas durante los veinte años que analizamos de la Capic, la Tabla 7 nos presenta los principales valores estadísticos. En particular, se observa como la Micro red 1, liderada por investigadores de la PUCV, posee el académico con más interacciones con sus cole- gas, esto es: 11 interacciones o grados-Maximun Degree-. Por su lado, el siguiente investigador que más interacciones de colaboración posee, se encontró en la Micro red 3, liderada en este caso por la UACh, con: 9 -Maximun Degree-. No obstante, a nivel general de interacciones o trabajos colaborativos entre investigadores de una mis- 
ma red, se aprecia que la Micro red 6, liderada por la UCSC, presentó el mejor estadístico, esto es: una densidad -Density- de 0,4222. En otras palabras, del total de posibles interacciones entre los 10 investigadores de la Micro red 6, se realizaron el $42,22 \%{ }^{5}$. Con respecto a otro indicador que aporta información en torno a la cohesión del grupo, se observa el diámetro de la red-Diameter-. En este sentido, la Micro red 1, así como la Micro red 2 poseen el mayor diámetro, esto es, seis. Mientras que las Micro redes: 3, 4, 5, 6 y
7, presentaron el menor valor, es decir, tres. Esto último significa una mayor probabilidad de interacción entre investigadores, debido a la cercanía entre ellos. No obstante, cabe destacar que las micro redes bajo análisis se han generado a través del tiempo, por ello, el mayor diámetro puede estar dado por situaciones particulares que afectaron a un investigador, por ejemplo, entre ellas tenemos el fallecimiento, la inactividad laboral, así como el retiro del campo académico por traslado al mundo empresarial.

Tabla 7. Propiedades estructurales de las ocho principales micro redes de colaboración académica en la Capic (1993-2015).

\begin{tabular}{|c|c|c|c|c|c|c|c|c|}
\hline \multirow{3}{*}{ Propiedades } & \multicolumn{8}{|c|}{ Denominación y valores de las principales micro redes } \\
\hline & Micro red 1 & Micro red 2 & Micro red 3 & Micro red 4 & Micro red 5 & Micro red 6 & Micro red 7 & Micro red 8 \\
\hline & PUCV & $\mathrm{UCN}$ & $\mathrm{UACh}$ & UDP & UMAG & UCSC & UST (COL) & UNAB \\
\hline A. TOTAL INVESTIGADORES & 24 & 17 & 14 & 13 & 12 & 10 & 9 & 7 \\
\hline \multicolumn{9}{|l|}{ B. NETWORK COHESION } \\
\hline Maximun Degree & 11 & 7 & 9 & 7 & 7 & 6 & 6 & 4 \\
\hline Minimun Degree & 1 & 1 & 1 & 1 & 1 & 2 & 1 & 1 \\
\hline Sum Degree & 88 & 48 & 58 & 38 & 38 & 38 & 24 & 16 \\
\hline Avg. Degree & 3,6667 & 2,8235 & 4,1429 & 2,9231 & 3,1667 & 3,8000 & 2,6667 & 2,2857 \\
\hline Std. Dev. Degree & 2,4438 & 1,4240 & 2,1665 & 1,6390 & 2,1148 & 1,3266 & 1,4907 & 1,0302 \\
\hline H-Index & 6,0000 & 3,0000 & 5,0000 & 3,0000 & 4,0000 & 4,0000 & 3,0000 & 3,0000 \\
\hline Density & 0,1594 & 0,1765 & 0,3187 & 0,2436 & 0,2879 & 0,4222 & 0,3333 & 0,3810 \\
\hline Components & 1 & 1 & 1 & 1 & 1 & 1 & 1 & 1 \\
\hline Component Ratio & 0,0000 & 0,0000 & 0,0000 & 0,0000 & 0,0000 & 0,0000 & 0,0000 & 0,0000 \\
\hline Connectedness & 1,0000 & 1,0000 & 1,0000 & 1,0000 & 1,0000 & 1,0000 & 1,0000 & 1,0000 \\
\hline Fragmentation & 0,0000 & 0,0000 & 0,0000 & 0,0000 & 0,0000 & 0,0000 & 0,0000 & 0,0000 \\
\hline Closure & 0,5238 & 0,4426 & 0,6532 & 0,4444 & 0,5294 & 0,6290 & 0,5000 & 0,4285 \\
\hline Avg. Distance & 2,7754 & 2,8309 & 1,9890 & 2,1410 & 1,9394 & 1,7778 & 1,9167 & 1,9524 \\
\hline SD. Distance & 1,2189 & 1,2459 & 0,7914 & 0,7799 & 0,7152 & 0,7569 & 0,7592 & 0,8985 \\
\hline Diameter & 6 & 6 & 3 & 3 & 3 & 3 & 3 & 4 \\
\hline Breadth & 0,5414 & 0,5408 & 0,3919 & 0,4423 & 0,3939 & 0,3222 & 0,3750 & 0,3611 \\
\hline Compactness & 0,4586 & 0,4592 & 0,6081 & 0,5577 & 0,6061 & 0,6778 & 0,6250 & 0,6389 \\
\hline
\end{tabular}

Fuente: elaboración propia en base a programa informático: Ucinet.

\section{CONCLUSIONES, RECOMENDACIONES E IMPLICANCIAS}

Las redes de colaboración académica se han transformado en una de las principales vías de fomento a la producción intelectual. En esta línea, la Conferencia Académica Permanente de Investigación Contable -Capic- ha construido durante su existencia un espacio de intercambio intelectual entre diversos académicos adscritos a universidades chilenas, tanto públicas como privadas. Es así como, durante más de dos décadas, la evidencia nos muestra que aproximadamente un $66 \%$ de los trabajos presentados a la Capic son elaborados bajo coautoría, predominando los trabajos colaborativos entre dos investigadores. No obstante, se observa una alta fragmentación de la red sociométrica, donde las principales micro redes o componentes se identifican con universidades específicas. En este aspecto, las principales redes se caracterizan por las relaciones de colaboración de naturaleza intrauniversitaria entre los investigadores.

Por otro lado, y en línea con lo indicado por Cárdenas (2016), se observa mayor cohesión en

5 Su cálculo se basa en dividir el total de interacciones reales -Sum Degree- por el total de posibles interacciones entre investigadores. En este sentido, el total de posibles interacciones para una red no dirigida o simétrica, corresponde a: [(número total de miembros de la red) * (número total de miembros de la red - 1)]. Para el caso de la Micro Red 6 es: [10*(10-1)]. 
las redes de Capic constituidas con menos de 20 integrantes. Sin embargo, existen algunos grupos de investigadores inactivos, es decir, no han participado en la Conferencia durante más de diez años, por el contrario se observan otros grupos consolidados de investigadores que participan constantemente en la Capic, en particular destaca la red formada por académicos de la Pontificia Universidad Católica de Valparaíso. Asimismo, durante el último tiempo se aprecia la constitución de una red internacional, en ella participan académicos de Colombia, Argentina y Chile. Además, otro patrón que se aprecia entre las principales redes al interior de la Conferencia se refiere a la movilidad laboral de ciertos investigadores, situación que favorece la ampliación del tamaño de la red.

Los resultados de esta investigación permiten visibilizar las principales estructuras de colaboración que se han generado a través del tiempo en el marco de la Conferencia anual. Lo anterior, puede facilitar a la sociedad científica Capic la definición de políticas de fomento a la producción intelectual. Sin embargo, es necesario realizar diversas acciones en torno a potenciar su institucionalidad, entre ellas recomendamos las siguientes: a) crear un repositorio, sea virtual o físico, para disponer de los artículos presentados a las conferencias; b) crear unos requerimientos mínimos de antecedentes a los autores, y a los artículos -metadatos-; lo anterior, con la finalidad de homogeneizar los datos, así como para acceder a mayor diversidad de atributos al momento de realizar análisis; c) generar una política formal de fomento a la producción académica, dando mayor énfasis a la conectividad extrauniversitaria, y en especial la incorporación de investigadores internacionales; y por último, d) fomentar el uso de las Tecnologías de la información y la comunicación (Tic).

Como implicancia de este trabajo se puede elaborar a futuro una investigación que permita caracterizar a los diversos investigadores, y por ende a las redes a las cuales pertenecen. Lo anterior, puede tomar como base el trabajo desarrollado por Cárdenas (2016), así como sustentarse en los conceptos de capital social, cultural, económico, político, y simbólico, acuñados por Bourdieu (1997, 2001). Esto con el propósito de identificar los diversos recursos que pueden ser compartidos entre los académicos de las diversas redes conformadas al alero de la Capic.

\section{REFERENCIAS BIBLIOGRÁFÍCAS}

Borgatti, S. (2002). NetDraw graph visualization software. Harveard: Analytic Technologies.

Borgatti, S., Everett, M., y Freeman, L. (2002). Ucinet 6 for Windows: Software for social network analysis. Harvard: Analytic Technologies.

Bourdieu, P. (1997). Capital cultural, escuela y espacio social. Siglo XXI, México.

Bourdieu, P. (2001). El capital social. Apuntes provisionales. Zona abierta, $\mathrm{n}^{\circ}$ 94-95, pp. 83-87.

Cárdenas, M. (2016). Sustentabilidad y redes de conocimiento. Análisis con la teoría de grafos. Publicaciones Empresariales UNAM FCA Publishing, México, distrito Federal.

Gaete, J., y Vásquez, J. (2008). Conocimiento y estructura en la investigación académica: una aproximación desde el análisis de redes sociales. REDES - Revista hispana para el análisis de redes sociales, 14(5), pp. 1-20.

González, I. (2008). Fragmento de la historia y protagonistas de Capic. Capic Review, n 6, pp. 1-7.

Olmeda, C., Perianes, A., y Ovalle, M. (2008). Estructura de las redes de colaboración científica entre las universidades españolas. Revista de sistemas de información y documentación, número monográfico, pp. 129-140.

Sanz, L. (2003). Análisis de redes sociales: o cómo representar las estructuras sociales subyacentes. Apuntes de Ciencia y Tecnologí, 7, 21-29.

Wasserman, S., y Faust, K. (2009). Social network analysis. Methods and applications. Cambridge University Press, New York.

Lu, H., y Feng, Y. (2008). A measure of authors' centrality in co-authorship networks based on the distribution of collaborative relationships. Scientometrics, 8(2), pp. 499-511. 